\title{
The rod and frame effect and induced head tilt as a function of observation distance
}

\author{
SHELDON M. EBENHOLTZ and TERRY L. BENZSCHAWEL \\ University of Wisconsin, Madison, Wisconsin 53706
}

\begin{abstract}
Several experiments investigated the influence of a tilted luminous frame on the rod and frame effect (RFE) and on adjustments of the head to apparent vertical, while observation distance to the frame was varied between 1 and $5 \mathrm{~m}$. Comparisons of repeated measures from the same subjects as well as between independent groups showed that distance was a highly effective variable. Both the RFE and the apparent head tilt varied inversely with distance to the frame, diminishing to zero at about $5 \mathrm{~m}$. Since head settings were in the direction of the tilted frame, the latter may be inferred to have caused an induced head tilt (IHT) opposite in direction to the tilted frame, when the head was objectively upright. Issues discussed were the role of IHT in the RFE and factors underlying the role of distance in both phenomena.
\end{abstract}

Since Witkin and Asch (1948) first investigated the effects of a tilted luminous frame on the apparent vertical orientation of a luminous line, a great number of studies on the rod and frame effect (RFE) have been reported (Long, 1974). However, a majority of these were designed for the purpose of correlational analysis and, as a consequence, they have not been concerned with the specification and manipulation of independent variables of which the RFE is a function. Although other studies have isolated many treatment variables affecting the RFE, including methodological factors (e.g., Beh, Wenderoth, \& Purcell, 1971; Howard \& Templeton, 1966; Wenderoth, 1974), witi a single exception (Gogel \& Newton, 1975), the possible role of observation distance has been overlooked. In their study, the focus of which was to determine the role of the relative depth interval between rod and frame, Gogel and Newton (1975) also examined the RFE in the coplanar configuration at distances of 1 and $1.6 \mathrm{~m}$. In one comparison, no significant distance effect occurred, but a second separate test, in which both frames were present simultaneously, showed that the farther frame yielded a significantly greater RFE than the nearer frame. It is not clear, however, to what extent the composite pattern created by the dual presence of the frames contributed to this outcome. Furthermore, since the far frame was set at $15 \mathrm{deg}$ CW with the near frame at $15 \mathrm{deg} \mathrm{CCW}$, the distances were totally confounded with the direction of frame tilt. Consequently, it is not possible to draw unambiguous conclusions about the role of distance.

This research was supported in part by NIMH Grant MH13006. Portions of the present paper were reported at the meetings of the Midwestern Psychological Association, Chicago, 1976.
Accordingly, the first purpose of the present study was to examine the effects of this potential determinant, namely, the distance between observer and frame, on the magnitude of the RFE.

The second purpose was to test the hypothesis that the tilted frame serves to induce an apparent head tilt in the subject that is opposite in direction to that of the frame ${ }^{1}$ (Brosgole \& Cristal, 1967; Templeton, 1973). Evidence for an induction effect could serve as the basis for an explanation of the RFE in terms of a processing algorithm (Ebenholtz, 1970, 1977), in which registered body or head tilt must be integrated with the optical input to yield the apparent orientation of a target. According to this view, a luminous line will be seen as vertical when its retinal image is oriented relative to the retinal vertica! meridian at an angle equal to the degree of registered head or body tilt, but opposite in direction. Thus, if a tilted frame induces head or body tilt opposite the frame tilt, the line would be set in the direction of frame tilt in order to appear upright, and this is precisely the direction required by the RFE.

Several previous studies have shown the postural vertical to be influenced by a tilted room (e.g., Passey, 1950; Witkin, 1949); however, since subjects had sight of portions of their own bodies as well as of the room, the results could reflect a purely visual conflict resulting in a "visual capture" of body position. The effect of a luminous frame on apparent head (or body) position in the absence of sight or self thus remains to be measured. Furthermore, a comparison of the effect of a tilted frame on apparent head position with its effect on the apparent vertical in the identical context, seems to have been neglected. Yet both should be influenced in similar ways by the same independent variables according to the induction-hypothesis. 


\section{EXPERIMENT 1}

\section{Method}

Procedure. In all the studies to be reported, the RFE was defined in terms of the algebraic difference in rod settings between frame-tilted and frame-upright conditions. This served to eliminate possible artifacts in the measurement of the RFE due to "bias" or constant error that might be present even with an upright frame. The choice of an upright frame as a control condition probably also worked to decrease the variance of the control settings relative to a procedure in which no frame was present (Gupta, 1973), but this should certainly not produce any systematic bias across the various experimental conditions. Finally it may be noted that the use of an upright frame permits a direct interpretation of any differences between upright and tiltedframe conditions as unambiguously attributable to the frame tilt and not to the presence of the frame as such.

The task was to set a luminous line to the apparent vertical with an upright or tilted luminous frame in the surround, and to position the head so that it felt upright while fixating a $2-\mathrm{cm}$ diam luminous dot seen at the center of an upright or tilted frame. All instructions were taped and the experimenter provided elaboration when requested. Both sets of instructions stressed the criterion of apparent upright with respect to the gravitational direction exemplified by the walls of the room. Subjects were urged not to pay attention to where the line or head actually was, but rather to set the line to where it looked upright and to adjust the head to where it felt upright.

Two line settings were made, one from each starting position at $30 \mathrm{deg} \mathrm{CW}$ and $\mathrm{CCW}$ of true vertical, and two head settings were taken, one from each starting position $45 \mathrm{deg}$ to the left and right shoulder, respectively.

Apparatus. The square frame was made of electroluminescent panels, $106.8 \mathrm{~cm}$ on a side and a width of $2.4 \mathrm{~cm}$. The line, which rotated around an axis $1.23 \mathrm{~m}$ above the floor at the center of the frame, had a luminous area with dimensions of $95.4 \mathrm{~cm} \times 2.2 \mathrm{~cm}$. Luminance of line and frame, respectively, was somewhat under 1 nit. The line rotated at a constant speed of $1 \mathrm{rpm}$ and its direction was controlled by the experimenter. The angular position of the line was registered on a meter with degree markings and was estimated to $.5 \mathrm{deg}$.

The subject was seated in a dental chair with the head placed in a brace that contained a chin cup and temple clamps. The entire brace was capable of rotating to the subject's left and right shoulder about an axis at the subject's neck and consequently permitted normal head movements during head settings to the postural vertical. These adjustments were read from a protractor fixed to the brace axis. After placement in the head restraint, the elevation of the dental chair was adjusted so that eye level conformed to the height of the line and frame axis, i.e., $1.23 \mathrm{~m}$. An electrically operated opaque curtain separated the subject from the rod and frame apparatus during adjustments of frame tilt and when readying the fixation spot for the head settings.

Design. Three groups of eight subjects each were exposed to the rod-and-frame apparatus at distances of $1,2.24$, and $5 \mathrm{~m}$ respectively, the values representing equal intervals on a $\log$ scale of distance. As distance increased, the retinal angle corresponding to one side of the frame decreased from 56.2 to 26.8 down to $12.2 \mathrm{deg}$ at the far distance. For all subjects, the frame was first set at 0 deg (upright), during which condition half the subjects in each group completed the two line settings followed by the two head adjustments to the apparent upright. The reverse task order was followed by the remaining subjects. After both head and line settings were taken, the identical sequence was repeated, but this time with the frame at $22 \mathrm{deg} \mathrm{CW}$. After all settings were completed with the frame at a given distance, all subjects repeated the line settings with frame at 0 and $22 \mathrm{deg}$, but with the frame placed at $2.24 \mathrm{~m}$. The data treated below are the differences in mean sellings, averaged over starling positions, between the tilted and upright frame conditions.

Subjects. After substituting one subject because of apparalus failure, a total of 24 subjects, 16 female, and 8 male, rook part. The subjects were assigned alternately to the three distance conditions according to order of appearance at the laboratory.

\section{Results and Discussion}

The results of the line and head settings at the three distances are represented in the first two rows of Table $1 .{ }^{2}$ There is a significant RFE $(p<.05)$ at the 1 and $2.24 \mathrm{~m}$ distances, but not at $5 \mathrm{~m}$. Furthermore, analysis of variance of the three groups yielded $F(2,21)=14.22, p<.01$, indicating that the RFE is sensitive to distance, the effect disappearing at $5 \mathrm{~m}$. A declining RFE with distance also was supported by a within-subjects comparison of performance at each subject's initial distance with that at $2.24 \mathrm{~m}$, the latter represented in the last row in Table 1. For the 1-m group, the RFE diminished significantly when tested at the 2.24-m distance, $\mathrm{t}(7)=3.26, \mathrm{p}<.01$, whereas there was no significant shift for the $2.24-\mathrm{m}$ group, as might be expected, $\mathrm{t}(7)=1.15, \mathrm{p}>.05$. On the other hand, the 5-m group showed a significant increase when tested at $2.24 \mathrm{~m}, \mathrm{t}(7)=3.42$, $\mathrm{p}<.01$, and thus the pattern of results is perfectly consistent with the independent groups analysis.

Head settings were not repeated for each subject at the 2.24-m distance, hence only independent group data are available, and these are represented in the second row of Table 1. Consistent with the line setting data, errors in head settings differed significantly from zero $(p<.05)$, but only at the 1 - and 2.24-m distances, and, as in the case of the RFE, the means differed significantly from one another, $\mathrm{F}(2,21)=6.27, \mathrm{p}<.01$.

The data consistently exhibit a RFE that varies inversely with observation distance. In addition, the adjustments of the head to the apparent upright yield errors in the direction of the tilted frame, thus supporting the hypothesis that the frame induces a tilt in the subject opposite the direction of the frame. This induced head tilt (IHT) also varied inversely with distance to the frame, and therefore the explanation of the RFE in terms of compensation for the IHT also was supported. However, since the magni-

Table 1

Error in Apparent Vertical (Degrees)

\begin{tabular}{|c|c|c|c|c|c|c|}
\hline \multirow[b]{3}{*}{ Task } & \multicolumn{6}{|c|}{ Distance to Frame } \\
\hline & \multicolumn{2}{|c|}{$1 \mathrm{~m}$} & \multicolumn{2}{|c|}{$2.24 \mathrm{~m}$} & \multicolumn{2}{|c|}{$5 \mathrm{~m}$} \\
\hline & $\mathbf{M}$ & $\sigma \mathrm{M}$ & $\mathbf{M}$ & $\sigma \mathbf{M}$ & $\mathbf{M}$ & $\sigma \mathrm{M}$ \\
\hline \multirow{3}{*}{$\begin{array}{l}\text { Line } \\
\text { Head }\end{array}$} & 7.57 & 1.11 & 4.66 & 1.33 & -.31 & .59 \\
\hline & 5.78 & 1.39 & 2.59 & .62 & -.91 & .78 \\
\hline & \multicolumn{6}{|c|}{ Control Task With Frame at $2.24 \mathrm{~m}$} \\
\hline Line & 4.93 & .97 & 5.66 & 1.18 & 1.16 & .65 \\
\hline
\end{tabular}

Note-Positive settings are in the direction of the tilted frame. 
tude of IHT was less than the RFE, the former could only represent a partial explanation of the latter.

Because of the large individual differences typically found in the RFE, between-subject comparison may be spurious for relatively small samples, and so provision was made in Experiment 1 for a series of within-subject comparisons as well, based upon line settings made by all subjects at $2.24 \mathrm{~m}$ after completing all previous settings. Such judgments are, however, necessarily confounded with possible sequence effects since they are preceded by settings taken at three different distances. Consequently, in Experiment 2, distance was treated as a within-subject variable and a statistical test (Grant, 1949) was made for possible sequence effects.

\section{EXPERIMENT 2}

\section{Design}

There were 28 student-volunteer subjects, 16 female and, 12 male, distributed among four conditions. All subjects were tested at two sessions placed 21 days apart in an effort to reduce any possible sequence (or transfer) effects of one session upon the subsequent session. The method of testing was the same as in the previous study with two exceptions. First, the line starting position was $20 \mathrm{deg} \mathrm{CW}$ and $\mathrm{CCW}$, and second, two settings were made at each starting position. In Condition 1-5, nine subjects made line and head settings to the apparent upright first at the $1-\mathrm{m}$ distance and then 21 days later with the frame at $5 \mathrm{~m}$. In Condition 5-1, nine additional subjects received the reverse sequence of distances. In Conditions $1-1$ and 5-5, five subjects each received the identical exposure distance of either 1 or $5 \mathrm{~m}$, respectively, at each session. The main purpose of these control conditions was to determine whether repetition of the test conditions over sessions might itsel! introduce a change in performance apart from any effects that might otherwise by attributable to the distance variable. With minor exceptions for the last four subjects, assignment to conditions alternated systematically among conditions 1-5, 5-1, and either $1-1$ or 5-5. At the second session, after the critical line and head settings had been taken, all subjects were tested with the frame at $2.24 \mathrm{~m}$.

\section{Results and Discussion}

The differences in line settings between the frametilted and frame-upright orientations, i.e., the RFE, are represented in Table 2 . In order to explore this pattern of results further, the two main conditions, $1-5$ and $5-1$, were analyzed to determine whether the order and sequence in which the distances were exposed and distance itself influence the magnitude of the RFE. Distance was highly significant, $F(1,16)$ $=20.01, \mathrm{p}<.01$, but there was a significant sequence effect as well, $F(1,16)=5.1, p<.05$. Since the latter was based upon an error term derived from the sum of squares for subjects (Grant, 1949), it may be concluded that the sequence 1-5 and 5-1 did indeed differ significantly beyond any effect due to subject differences. The nature of the effect is that the second task is influenced in the direction of the first, a rather surprising outcome given the 21 -day interval between sessions. Because of these sequence effects,
Table 2

Error in Apparent Vertical for Line Settings (Degrees)

\begin{tabular}{|c|c|c|c|c|c|c|c|}
\hline \multirow[b]{3}{*}{ Condition } & \multicolumn{6}{|c|}{ Distance to Frame } & \multirow[b]{3}{*}{$\mathrm{n}$} \\
\hline & \multicolumn{2}{|c|}{$1 \mathrm{~m}$} & \multicolumn{2}{|c|}{$5 \mathrm{~m}$} & \multicolumn{2}{|c|}{$2.24 \mathrm{~m}$} & \\
\hline & $\mathbf{M}$ & $\sigma \mathrm{M}$ & $\mathbf{M}$ & $\sigma \mathbf{M}$ & $\mathbf{M}$ & $\sigma \mathbf{M}$ & \\
\hline $1-5$ & 8.60 & 1.54 & 4.84 & 1.15 & 5.78 & 1.34 & 9 \\
\hline $5-1$ & 4.99 & 1.25 & 1.20 & 1.15 & 3.17 & 1.00 & 9 \\
\hline $1-1$ & $\begin{array}{l}5.04 \\
5.20\end{array}$ & $\begin{array}{r}1.71 \\
.99\end{array}$ & & & 2.88 & 1.27 & 5 \\
\hline $5-5$ & & & $\begin{array}{l}-.16 \\
-.12\end{array}$ & $\begin{array}{l}.39 \\
.42\end{array}$ & .82 & .48 & 5 \\
\hline
\end{tabular}

Note-Positive settings are in the direction of the tilted frame. The upper member of the pair of entries for Conditions 1-1 and 5-5 represents the first distance to which these subjects were exposed.

each condition had to be analyzed separately for the possible effects of distance. In both conditions 1-5 and condition 5-1, the differences between the $1-\mathrm{m}$ and $5-\mathrm{m}$ distances were significant, in favor of the former, $\mathrm{t}(8)=2.71, \mathrm{p}<.02$, and $\mathrm{t}(8)=3.99$, $p<.01$, respectively. The inverse relation between distance and the RFE found in Experiment 1 is thus confirmed.

The control conditions (1-1 and 5-5) showed no evidence at all of any change due to the repetition of the identical task over the 21-day period. This outcome may reflect the same sequence effect described above where performance on the second task moved in the direction of the first, which in this case would work to insure identical performance at both sessions, as obtained. In the absence of a difference between sessions, the control data were pooled and mean performance at $1 \mathrm{~m}$ was compared with that at $5 \mathrm{~m}$ (viz., condition 1-1 vs. condition 5-5). Again, the difference was significant, in favor of the nearer frame, $\mathrm{t}(8)=3.85, \mathrm{p}<.01$.

The data in the right-hand column at Table 2, taken at 2.24, represent settings made at the second session, after all other data had been collected. It is highly likely, therefore, that they reflect sequence effects not only as a result of the first session but of the preceding settings of the same day as well. Consequently, no statistical analyses will be undertaken with these data, although it may be pointed out that in each condition the ordinal position of the mean at $2.24 \mathrm{~m}$ is appropriate to the hypothesis, viz., performance at $2.24 \mathrm{~m}$ falls between that at 1 and $5 \mathrm{~m}$, is less than that at $1 \mathrm{~m}$ for condition $1-1$ and is greater than that at $5 \mathrm{~m}$ for condition 5-5.

Head settings, representing the mean difference in performance between frame-upright and frametilted conditions, are represented in Table 3. At the $1-\mathrm{m}$ distance, only the mean of the second session of condition 1-1 failed to be significantly different from zero at $\mathbf{p}=.05$ or better. In contrast, at $5 \mathrm{~m}$, the only significant effect occurred in condition 1-5, 
where settings were preceded by the $1-m$ condition 21 days earlier. With minor exception, this pattern of induced head-tilt effects is remarkable similar to the pattern of the RFE already described. Analysis of variance for order, sequence, and distance, made on the data of conditions 1-5 and 5-1 yielded a significant effect of distance, $F(1,16)=26.38, p<.01$, but, as in the case of the RFE data, a sequence effect emerged that was significant beyond any effect attributable to subject differences in the two conditions $F(1,16)=4.53, p<.05$. Again, the sequence effect worked to modify the settings in the direction of those of the previous session. Separate (withinsubject) comparisons for each condition yielded significant differences for condition $1-5, t(8)=3.17$, $\mathrm{p}<.01$, and condition $5-1, \mathrm{t}(8)=5.14, \mathrm{p}<.01$. In each case, induction effects were greater, the nearer the frame.

Just as in the case of the RFE data, control conditions $1-1$ and 5-5 showed no significant changes from one session to the next. The data were, therefore, combined over sessions and compared for distance effects. These differences did not, however, reach significance, $\mathrm{t}(8)=1.66, \mathrm{p}>.05$.

The final settings, taken at $2.24 \mathrm{~m}$, were not analyzed because of the strong possibility of sequence effects. However, note may be taken of the fact that the obtained means all fall into the proper ordinal relationship with the data at 1 and $5 \mathrm{~m}$, respectively. The effect of distance on induced head tilt is thus remarkably similar to its effect on the RFE, although the magnitude of the former at maximum is considerably less than that of the latter.

The relationships between the RFE and induced head tilt and of the two to distance are summarized in Figure 1, where, together with the results of the two experiments already described, there is represented the data of two additional groups with the frame at $3 \mathrm{~m}$, producing a retinal angle of $20.2 \mathrm{deg}$. These two groups (16 subjects in one, 8 in the other) were constituted of the same subject population as that of those already reported, and they. received instructions and treatment identical to those administered the subjects of Experiments 1 and 2. The only exceptions were the use of line starting positions at $30 \mathrm{deg} \mathrm{CW}$ and $\mathrm{CCW}$ and of head starting positions at $45 \mathrm{deg}$ on either side of vertical. In all groups, the same subjects contributed both line and head settings, but each subject was tested at only one distance. Thus, the data points represent nine independent groups and are those of the first distance to which the subject was exposed, thus eliminating the possible influence of prior settings. In summary, the subjects in all nine groups represented in Figure 1 were treated virtually identically, with minor exception for the number of settings at each starting position (one or two) and slight differences in the magnitude of the starting position of line and head. The
Table 3

Error in Apparent Vertical for Head Settings (Degrees)

\begin{tabular}{|c|c|c|c|c|c|c|c|}
\hline \multirow[b]{3}{*}{ Condition } & \multicolumn{6}{|c|}{ Distance to Frame } & \multirow[b]{3}{*}{$\mathrm{n}$} \\
\hline & \multicolumn{2}{|c|}{$1 \mathrm{~m}$} & \multicolumn{2}{|c|}{$5 \mathrm{~m}$} & \multicolumn{2}{|c|}{$2.24 \mathrm{~m}$} & \\
\hline & $\mathbf{M}$ & $\sigma \mathbf{M}$ & $\mathbf{M}$ & $\sigma \mathbf{M}$ & $\mathbf{M}$ & $\sigma \mathrm{M}$ & \\
\hline $1-5$ & 3.48 & .84 & 1.26 & .57 & 1.79 & .82 & 9 \\
\hline $5-1$ & 1.84 & .24 & .04 & .33 & 1.08 & .42 & 9 \\
\hline $1-1$ & $\begin{array}{l}1.80 \\
1.88\end{array}$ & $\begin{array}{r}.84 \\
1.45\end{array}$ & & & .46 & 1.05 & 5 \\
\hline $5-5$ & & & $\begin{array}{r}-.12 \\
.02\end{array}$ & $\begin{array}{l}.64 \\
.42\end{array}$ & .68 & .76 & 5 \\
\hline
\end{tabular}

Note-Positive settings are in the direction of the tilted frame. The upper member of the pair of entries for Conditions I-I and 5-5 represents the first distance to which these subjects were exposed.

solid lines in Figure 1 connect the means of the data points at each distance weighted in terms of group size. Both functions are quite linear, converge to zero at about $5 \mathrm{~m}$, and exhibit highly similar effects of distance. This mutual dependency is also expressed in the significant correlation between the nine pairs of head-setting and line-setting points $\varrho(9)=$ $.867, \mathrm{p}<.01$.

\section{GENERAL DISCUSSION}

The RFE has been shown to be dependent upon distance, but just why this should be so has not been addressed. The possibilities are that the critical vari-

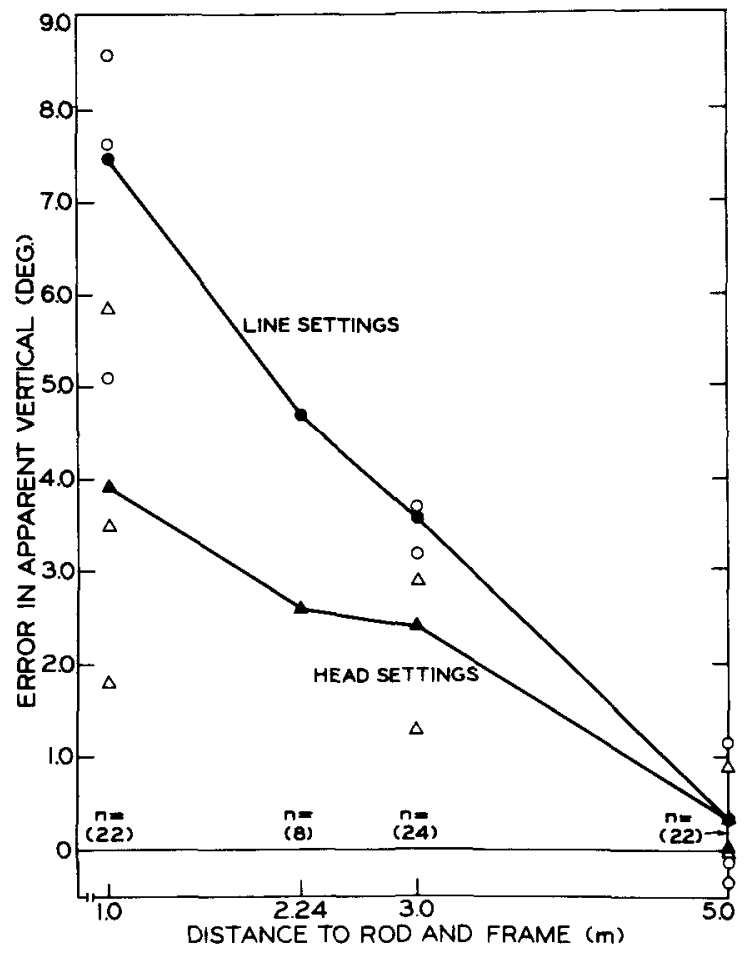

Figure 1. Error in apparent vertical as a function of distance to the rod and frame. 
able is apparent distance or that distance merely serves 10 mediate other factors such as apparent and/or retinal size. These remain to be investigated in subsequent studies. ${ }^{3}$

It is widely known that performance in the rodand-frame task differs greatly among individuals, and considerable intersubject variation was recorded in the present studies. Whatever factors are responsible for these individual differences, they must, on the basis of the present results, be thought of in terms of interactions with distance for at the nearest distance where the RFE is maximal the greatest variation among individual scores is permitted. On the other hand, at great distances, the RFE moves toward zero and there is, as a consequence, little room for individual differences to be exhibited. Accordingly, if one is to find differential performance in terms of, for example, field dependence and field independence, there is a greater likelihood of so doing at near rather than at far distances to the frame. There is in this a potential source of uncontrolled variation, especially in crossexperimental comparisons. But, more importantly, factors proposed to account for individual differences, such as cognitive style (e.g., Witkin \& Oltman, 1967), must be interpreted in light of their interaction with distance or factors mediated by distance, such as retinal size or retinal locus.

The influence of a tilted frame on apparent head tilt was such as to require the subject to move the head in the direction of the frame in order for it to feel upright. By implication, when the head was maintained truly upright, as when making line settings, there occurred an induced head tilt opposite the direction of the frame. On the premise that apparent orientation is determined by a processing algorithm that is a joint function of registered head tilt and the retinal orientation of the line (Ebenholtz, $1970,1977)$, the latter would have to be rotated opposite the direction of registered head tilt in order to correspond with an apparently upright target. Since this is precisely the direction required to account for the RFE, it may be concluded that induced head tilt is, in fact, a contributor to the RFE. That it is not the only cause of the RFE follows from the fact that the function for induced head tilt was consistently lower than the RFE function. It is possible that the method of measurement, i.e., having the subject rotate his head to the apparent upright, may, by providing kinaesthetic feedback, have produced a conservative measure. A similarly conservative outcome could have resulted from the changing retinal pattern during head adjustments, a situation amounting to an induction stimulus of varying magnitude because of the changing retinal tilt of the frame. Nevertheless, it remains unlikely that the RFE is caused exclusively by induced head tilt, because the effect can be obtained with the subject in a supine orientation (Rock, 1975). In this posture, setting a line to an egocentric reference such as the median sagitral axis is influenced by the presence of a tilted frame, as in the more typical upright position. But an induced head tilt could have no effect in the supine posture, since the required orientation is defined with reference to an axis on the head itself. Rock (1975) has suggested that the tilted frame may cause a shift in egocentric orientation of a line with respect to the sagittal axis of the head, even when the subject is upright, and this may represent the second factor which, along with induced head tilt, combines to produce the RFE. ${ }^{4}$

It is important to note that in the present studies induced head tilt was purely a function of the presentation of the tilted luminous frame in an otherwise completely dark environment. It represents, therefore, a direct effect of a visible target on proprioception, ${ }^{5}$ a condition different from previous cases in which sight of a tilted room was shown to influence the postural vertical by conflicting with sight of the body (e.g., Witkin, 1949). The present findings are more directly comparable to the effects of a rotating pattern on both object and body orientation (Held, Dichgans, \& Bauer, 1975), with the qualification that the induction effects reported herein were based upon static displays. If these inducing conditions are indeed analogous, then they may share a common cause in the relatively greater effectiveness of peripheral, as opposed to foveal, stimulation found with moving displays. The reason for the diminished induction and line-orientation effects with increased frame distance would then follow as a logical consequence of the shift in position of retinal pattern from peripheral to foveal stimulation with increasing distance.

\section{REFERENCES}

Beh, H. C., Wenderoth. P. M., \& Purcell, A. T. The angular function of a rod-and-frame illusion. Perception \& Psychophysics, 1971, 9. 353-355.

Brosgole, L..\& CRISTAL. R. M. The role of phenomenal displacement on the perception of the visual upright. Perception \& Psychophysics. 1967. 2. 179-188.

Ebenholtz. S. M. Perception of the vertical with budy tilt in the median plane. Joumal of Experimental Psychology, 1970, 83. $1-0$.

Eвenholtz. S. M. The constancies in object orientation: An algorithm processing approach. In W. Epstein (Ed.). Stability and constancy in visual perception: Mechanisms and processes. New York: Wiley, 1977.

Gogel. W. C.. \& Newton, R. E. Depth adjacency and the rodand-fiame illusion. Perception \& Psychophysics, 1975, 18. 163-171.

Grant, D. A. The statistical analysis of a frequent experimental design. American Journal of Psychology, 1949, 62. 119-122.

Gupta, G. C. Effect of lateral body tilts and visual frames on perception of the apparent vertical. Journal of Experimental Psychology, 1973. 100, 162-167. 
Held, R., Dichgans. J.. \& Bauer, J. Characteristics of moving visual scenes influencing spatial orientation. Vision Research. 1975. 15. 357-365.

Howard. 1. P.. d Templeton, W. B. Human spatial orientation. New York: Wiley. 1966.

KoffKa. K. Principles of gestalt psychology. New York: Harcourt. Brace. 1935

LonG. G. M. Reported correlates of perceptual style: A review of the field dependency-independency dimension. Journal Supplement Abstract Service Catalog of Selected Documents in Psychology, 1974, 4, 1-82, Ms. No. 540.

PASSEY. G. E. The perception of the vertical. IV. Adjustment to the vertical with normal and tilted visual frames of reference. Journal of Experimental Psychology. 1950, 40, 738-745.

Rock. 1. An introduction to perception. New York: Macmillan. 1975.

TEMPLETon, W. B. The role of gravitational cues in the judgment of visual orientation. Perception \& Psychophysics, 1973, 14. 451.457.

WENDEROTH, P. M. The distinction between the rod-and-frame illusion and the rod-and-frame test. Perception, 1974, 3. 205-212.

WITKIN. H. A. Perception of body position and of the position of the field. Psychological Monographs, 1949, 63(7. Whole No. 302).

WITKIN. H. A., \& Asch, S. E. Studies in space orientation. IV. Further experiments on perception of the upright with displaced visual fields. Journal of Experimental Psychology. 1948. 38. 762-782.

Witkin. H. A.. \& Oltman, P. K. Cognitive style. International Journal of Neurology, 1967. 6. 119-137.

\section{VOTES}

1. In somewhat different terms, this expresses Koffka's (1935) conception of the importance of the framework in orientation perception, viz., "both the Ego and the objects within the framework are determined by the framework and its invariant connections with its content, viz.. objects and Ego" (p. 221).

2. For one subject, line settings averaged 19.25 and $20.25 \mathrm{deg}$ at 1 and $2.24 \mathrm{~m}$, respectively, the most extreme scores in the entire study. In order to reduce the inordinate contribution to variance of these scores, the mean of the remaining seven subjects was substituted.

3. A report is now in preparation supporting the role of retinal size as a determinant of the RFE.

4. It does not necessarily follow that, in the absence of the component attributable to induced head tilt, the RFE should decrease in the supine position relative to the upright, since the effectiveness of the egocentric component itself probably varies as a function of body posture.

5. There are several potential sources of proprioception for head position. Among these are the otolith organs, joint receptors in the cervical vertebrae, neck-muscle spindles, and perhaps even the semicircular canals themselves.

(Received for publication April 7, 1977; revision accepted July 28,1977 .) 